\title{
HGSA Abstract
}

\section{Poster Presentation From the 40th Human Genetics Society of Australasia Annual Scientific Meeting}

\author{
This poster was inadvertently omitted from the HGSA \\ abstracts published in 2016 in Twin Research and Human \\ Genetics, volume 19, issue no. 5.
}

\section{${ }^{I}$ Universiti Sains Malaysia, Kelantan, Malaysia \\ ${ }^{2}$ International Medical University, Kuala Lumpur, Malaysia \\ ${ }^{3}$ Universiti Teknologi MARA, Perak, Malaysia}

2. STATUS OF TELOMERASE COMPONENTS (TERT AND TERC GENES) IN RELATION WITH IMATINIB MESYLATE RESISTANCE IN CHRONIC MYELOID LEUKAEMIA PATIENTS

Sarina Sulong,' Wati Hayati Shamshudin,' Rosline Hassan,' Azlan Husin,' Abdul Aziz Baba, ${ }^{2}$ Zaidatul Shakila Mohamad Ashari, ${ }^{3}$ and Ravindran Ankathil

Imatinib mesylate (IM) has been used as a first line therapy in chronic myeloid leukaemia (CML) patients since 2001. Besides a successful treatment for majority of patients, about one-third of patients were reported to be resistant upon this drug. Direct association of telomerase activation has been observed in cancer cells. However, the mechanism of resistance remains inconclusive. Telomerase is a ribonucleoprotein enzyme having two core components; a catalytic reverse transcriptase protein unit, TERT, and an RNA subunit, TERC. To determine the possible contribution of telomerase core components in relation with IM resistance, we evaluated $79 \mathrm{CML}$ samples $(52=$ good respond, $27=$ resistant $)$ with 62 (chronic), 6 (accelerated), 1 (blastic), and 10 samples having no information on disease phase. Patients were recruited from Hospital USM and other government hospitals in Malaysia. The expression levels of TERT and the copy number of TERC in both resistant and respond groups were determined and analyzed by two-tailed Mann-Whitney $U$ test and $t$ test. Both TERT expression and TERC copies showed no significant differences between resistant and respond groups $(p=.122$ and $p=.061$, respectively). However, we found a patient who previously responded to IM at chronic phase but later became resistant after entering blastic phase with 11 times higher reading of fold difference of hTERT expression. This situation may support a reported study showing that over-expression of hTERT favors the development of IM resistance. Further study with a large sample size is warranted to elucidate the role of telomerase components in IM resistance. 